\title{
Q.
QNEEN'S
UNIVERSITY
BELFAST
}

\section{Small-Signal Refinement of Power System Static Load Modelling Techniques}

McLorn, G., \& McLoone, S. (2017). Small-Signal Refinement of Power System Static Load Modelling

Techniques. In Advanced Computational Methods in Energy, Power, Electric Vehicles, and Their Integration International Conference on Life System Modeling and Simulation, LSMS 2017 and International Conference on Intelligent Computing for Sustainable Energy and Environment, ICSEE 2017, Proceedings (Vol. 763, pp. 467476). (Communications in Computer and Information Science; Vol. 763). Springer Verlag. https://doi.org/10.1007/978-981-10-6364-0_47

Published in:

Advanced Computational Methods in Energy, Power, Electric Vehicles, and Their Integration - International Conference on Life System Modeling and Simulation, LSMS 2017 and International Conference on Intelligent Computing for Sustainable Energy and Environment, ICSEE 2017, Proceedings

\section{Document Version:}

Peer reviewed version

Queen's University Belfast - Research Portal:

Link to publication record in Queen's University Belfast Research Portal

\section{Publisher rights}

(C) 2017 Springer International Publishing AG.

This work is made available online in accordance with the publisher's policies. Please refer to any applicable terms of use of the publisher.

\section{General rights}

Copyright for the publications made accessible via the Queen's University Belfast Research Portal is retained by the author(s) and / or other copyright owners and it is a condition of accessing these publications that users recognise and abide by the legal requirements associated with these rights.

\section{Take down policy}

The Research Portal is Queen's institutional repository that provides access to Queen's research output. Every effort has been made to ensure that content in the Research Portal does not infringe any person's rights, or applicable UK laws. If you discover content in the Research Portal that you believe breaches copyright or violates any law, please contact openaccess@qub.ac.uk. 


\title{
Small-Signal Refinement of Power System Static Load Modelling Techniques
}

\author{
Gareth McLorn ${ }^{1}$ and Seán McLoone ${ }^{1}$ \\ ${ }^{1}$ School of Electronics, Electrical Engineering and Computer Science, \\ Queen's University Belfast, Belfast, Northern Ireland, U.K. \\ gmclorn02@qub.ac.uk, s.mcloone@qub.ac.uk
}

\begin{abstract}
Loads are often represented as a weighted combination of constant impedance (Z), current (I) and power (P) components, so called ZIP models, by various power systems network simulation tools. However, with the growing need to model nonlinear load types, such as LED lighting, ZIP models are increasingly rendered inadequate in fully representing the voltage dependency of power consumption traits. In this paper we propose the use of small-signal ZIP models, derived from a neural network model of appliance level consumption profiles, to enable better characterizations of voltage dependent load behavior. Direct and indirect approaches to small-signal ZIP model parameter estimation are presented, with the latter method shown to be the most robust to neural network approximation errors. The proposed methodology is demonstrated using both simulation and experimentally collected load data.
\end{abstract}

Keywords: ZIP models, exponential models, load modelling, neural networks.

\section{Introduction}

This paper proposes an enhancement of existing static load modelling techniques to facilitate greater accuracy in the characterization of active and reactive power consumption, as functions of applied voltage, in power systems studies. This research is pertinent, given the emergence of many modern load types for which conventionally understood behaviors, in response to imposed variations in voltage, do not readily apply. Conventional modelling practices ought to be reviewed so that the characteristics of modern loads are reproduced in simulation with greater fidelity and their aggregated influence upon electricity networks may become better quantified.

Conservation Voltage Reduction (CVR) is a noteworthy energy conservation application, for which the accuracy of load models is intrinsic to its effectiveness. Utilities tend to employ CVR to lower service level voltages as a proxy for reducing electricity demand, especially during peak hours. Its objectives are typically achieved via the coordinated control of assorted distribution network assets, such as on load tapchanging (OLTC) transformers and switched capacitor banks. The effectiveness of enacting CVR within a targeted electricity network may be encapsulated by its CVR factor [1], values which aim to quantify the responsiveness (in percentage terms) of the energy consumed by an electrical appliance, household, feeder or entire network, $\Delta E$, to an applied percentage change in the voltage supply, $\Delta V$. For changes in active energy, 
this paper qualifies the corresponding CVR factor scalar with a 'p' subscript, as in $C V R_{p}$. Similarly, for reactive power the notation $C V R_{q}$ is used. In the medium to long term the $C V R_{p}$ scalar may be used to predict the total energy savings attributable to CVR. In the short term steady-state, it may also be used by utilities to predict the demand reduction impact of CVR during the daily peak [2]-[3].

$$
C V R_{p}=\frac{\Delta E(\%)}{\Delta V(\%)}
$$

$C V R_{p}$ ratios are in effect concise, aggregated representations of the steady-state parameters that individual loads present to the network upon connection. Ultimately, it is the aggregated combination of the many different loads within each household (modelled as a lumped load) and the composite $C V R_{p}$ quantity therein that will determine the extent by which customers save energy (if at all) within a CVR scheme. The aggregation concept may be extended further so that CVR factors determined at the appliance, customer and feeder levels are used to form a CVR factor for the entire network. Other research [4]-[5] has sought to overcome the complexity of extrapolating a deluge of individual load traits to the network level by introducing a linearized approximation to the established definition for $C V R_{p}$ outlined in (1).

This paper explores new ways of interpreting load behaviors in the presence of voltage fluctuations, beyond that of established modelling practice, with the intention of assisting the analysis and predictability of network voltage optimization tools, such as CVR. Specifically, a small-signal load characterization methodology is proposed in which an accurate nonlinear model of the load behavior is first generated using a Multilayer Perceptron (MLP) neural network, and then voltage dependent small-signal load model parameters derived analytically as function of the MLP parameters.

The remainder of the paper is organized as follows. Section 2 introduces the two most widely used static load models (ZIP and exponential), and discusses their weaknesses. The proposed small-signal neural network based methodology is then described in Section 3. Results demonstrating the efficacy of the methodology are presented in Section 4 and finally Section 5 concludes the paper.

\section{$2 \quad$ Static Load Modelling}

\subsection{ZIP models}

The ZIP load modelling approach [7] seeks to approximate active or reactive power draw as a function of applied voltage, expressed in the form of a quadratic polynomial. The coefficients of the model correspond to constant impedance $(Z)$, constant current (I) and constant power (P) consumption terms. An equality constraint applies to the model, whereby the values of the three coefficients must exactly sum to 1 . The ZIP formulation for active power, $P(V)$, is given by the following equation, in which $P_{0}$ corresponds to the active power observed at the nominal voltage level, $V_{0}$.

$$
P(V)=P_{0} \cdot\left[Z_{p} \cdot\left(\frac{V}{V_{0}}\right)^{2}+I_{p} \cdot\left(\frac{V}{V_{0}}\right)+P_{p}\right]
$$


Cursory application of (2) shows that for a purely resistive load, i.e. one for which $Z_{p}=1, I_{p}=0$ and $P_{p}=0$, the active power consumption scales with the square of the applied voltage level. Thus, the application of CVR to such loads is highly conducive in terms of reducing the power consumption of end users. The classic interpretation of a constant impedance load is the incandescent lamp [6], which until very recent times was a ubiquitous feature of distribution networks and a major component of aggregate demand. Conversely, for purely constant power loads, i.e. where $Z_{p}=0, I_{p}=0$ and $P_{p}$ $=1$, active power consumption is wholly independent of applied voltage fluctuations.

Load types of this nature are not expected to benefit from the application of CVR. Moreover, the act of reducing the voltages applied to such devices is, by definition, consistent with increasing the currents drawn and so the impact of CVR may be to increase the level of network losses incurred in delivering power to end users.

Given some measured data sets, $\mathbf{P}$ and $\mathbf{V}$, obtained for a load type that is subject to characterization, two approaches exist to estimate the resulting ZIP model:

$$
\mathbf{P}=\left[\begin{array}{llll}
P_{1} & P_{2} & \cdots & P_{m}
\end{array}\right]^{\mathrm{T}}, \quad \mathbf{V}=\left[\begin{array}{lllll}
V_{1} & V_{2} & \cdots & V_{m}
\end{array}\right]^{\mathrm{T}},
$$

where $m$ is the number of measurements (data points). The first step is to convert the data to per-unit form (i.e. $\mathbf{P}_{\mathrm{pu}}=\mathbf{P} / P_{0}, \mathbf{V}_{\mathrm{pu}}=\mathbf{V} / V_{0}$ ) and to then employ constrained least squares regression to estimate the ZIP parameters [9]. This method assumes that the nominal power $\left(P_{0}\right)$ is known a priori. The second approach fits a quadratic model to the raw data. From this the nominal power $\left(P_{0}\right)$ level corresponding to the nominal voltage $\left(V_{0}\right)$ is derived and the corresponding per-unit ZIP parameters can be deduced analytically. The advantage of the latter approach is that the sum to unity constraint on ZIP parameters, outlined in [8], is inherently satisfied. Hence, parameter estimation can be performed via conventional unconstrained least squares regression.

\section{$2.2 \quad$ Exponential models}

The exponential load model approximates the load power-voltage curve as

$$
P(V)=P_{0} \cdot\left(\frac{V}{V_{0}}\right)^{n_{p}}
$$

In contrast to the ZIP model, in (4) a load's relationship with voltage is encapsulated by a single exponent term, $n_{p}$, rather than three individual scalars. For small variations in applied voltage, it is possible to show, algebraically, that $n_{p}$ offers a reasonable approximation to $C V R_{p}$. Synergies with the ZIP model become apparent by once again considering the attributes of constant impedance and constant power example load types. For a constant impedance load, (2) and (4) are observed to be equivalent when $n_{p}=2$; whereas for constant power loads, $n_{p}=0$. More generally, by computing a quadratic approximation to the exponential model at the nominal voltage, the relationship between the ZIP and exponential parameters can be expressed as

$$
Z_{p}=\frac{1}{2} n_{p}\left(n_{p}-1\right), I_{p}=n_{p}\left(2-n_{p}\right), \text { and } P_{p}=\frac{1}{2}\left(n_{p}^{2}-3 n_{p}+2\right)
$$

Therefore, each $n_{p}$ maps to an equivalent set of ZIP parameters. 
The exponential model can also be estimated from measured data, taken in either raw or per unit form. A linear least squares estimate can be obtained by taking the log of per-unit data, giving

$$
n_{p}=\frac{\left[\log \left(\mathbf{V}_{\mathrm{pu}}\right)\right]^{\mathrm{T}}}{\left[\log \left(\mathbf{V}_{\mathrm{pu}}\right)\right]^{\mathrm{T}}} \frac{\left[\log \left(\mathbf{P}_{\mathrm{pu}}\right)\right]}{\left[\log \left(\mathbf{V}_{\mathrm{pu}}\right)\right]}
$$

When working with raw data, expressed in base units rather than per unit, both $P_{0}$ and $\mathrm{n}_{p}$ must be estimated. Again logarithms are taken, which allows the parameter estimates to obtained from $n_{p}=\theta_{1}$ and $P_{0}=\exp \left(\theta_{2}\right)$, where $\mathbf{X}^{\dagger}$ denotes the MoorePenrose pseudoinverse of $\mathbf{X}$, and

$$
\boldsymbol{\theta}=\left[\begin{array}{l}
\theta_{1} \\
\theta_{2}
\end{array}\right]=\left[\begin{array}{ll}
\log (\mathbf{V}) & \mathbf{1}
\end{array}\right]^{\dagger}[\log (\mathbf{P})]
$$

\subsection{Load Modelling Challenges}

The application of both the ZIP and exponential load models is best suited to the characterization of those load types that exhibit simple relationships with applied voltage. As such, their ongoing applicability has arguably become more dubious as the adoption of complex, power electronic circuitry within everyday consumer appliances has proliferated. Many such devices exhibit strongly non-linear or piecewise voltage response characteristics, against which the quadratic composition of the classic ZIP load model (2) can often only muster a rudimentary fit.

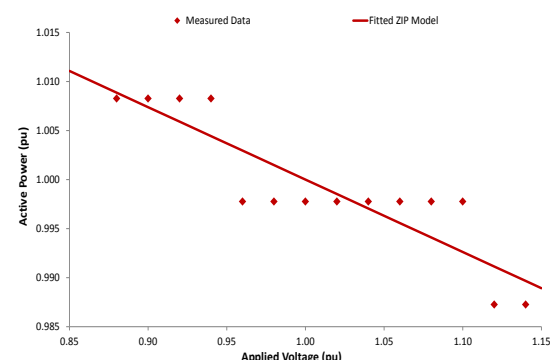

(a) LED light bulb

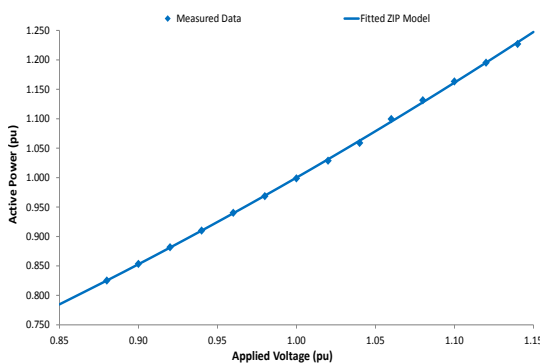

(b) Incandescent light bulb

Fig. 1: Per-unit active power consumption profile versus per-unit applied voltage, for: (a) a modern $9 \mathrm{~W}$ LED rated lamp, and (b) a traditional $60 \mathrm{~W}$ rated incandescent lamp

An example of piecewise active power consumption behavior is presented in Fig. 1(a) for a modern, Light Emitting Diode (LED) based lamp. Lamp performance has been tested under strict laboratory conditions across a range of applied voltage levels within the statutory range. The ensuing active power characteristic is not readily resolved to a quadratic function through ZIP modelling techniques. As such, the equivalent ZIPfitted curve (depicted by the continuous line trace) resolves to a linearly sloping power against voltage relationship, across all voltage levels. Conversely, the raw measured data (depicted by the scattered points) portrays a piecewise, constant power trend, within which different constant power levels are defined across different bands of applied voltage. A similar characteristic, across the same range of voltages, is presented 
for an incandescent bulb in Fig. 1(b). In this instance the ZIP-fitted curve offers a much better approximation to the measured data.

The divergent characteristics exhibited for the LED lamps are indicative of why new innovations in load modelling are increasingly required, as the limitations of fitting modeled behaviors to simplistic quadratic or exponential functions becomes more glaring within network level, power systems studies. Many load flow simulation tools invoke ZIP load modelling techniques. Amongst the most compelling arguments for continuing this approach is the convention that each of the ZIP coefficients, $Z_{p}, I_{p}$ and $\mathrm{P}_{\mathrm{p}}$, pertain to a physical property (impedance, current and power) that is easy for electrical engineers to comprehend and thus convenient for analysis. However, aside from potential increases in computational effort, there are few clear justifications for precluding the use more complex models to represent the load characteristics in simulation, especially if they are able to impart greater levels of accuracy.

\section{Small-Signal Neural Network Based Load Modelling}

As discussed previously, the use of a stationary set of ZIP or exponential parameters to model load characteristics, when fitted across a relatively wide range of voltage variations, fails to render a precise characterization for many modern loads. We propose an alternative framework, in which model coefficients are resolved dynamically with respect to the prevailing voltage level, across the full range of applied voltage. This approach ensures that a higher level of accuracy is obtained, as the characteristic can be more readily fitted against localized perturbations and piecewise functionality. A general nonlinear modelling paradigm $\hat{P}=f(V, \mathbf{W})$ is employed, where the model parameters, $\mathbf{W}$, are chosen so as to minimize $\|\mathbf{P}-\widehat{\mathbf{P}}\|_{2}^{2}$. From a Taylor series expansion of $f(V, \mathbf{W})$, small-signal ZIP and exponential models are derived to describe load behaviors with respect to small changes in voltage, about a localized reference point. The small-signal exponential model can be obtained as

$$
n_{p}(V)=V \frac{f^{\prime}(V, \mathbf{W})}{f(V, \mathbf{W})}
$$

and the corresponding small signal ZIP parameters are given by

$$
\begin{gathered}
Z_{p}(V)=\frac{V^{2} f^{\prime \prime}(V, \mathbf{W})}{2 f(V, \mathbf{W})}, \quad I_{p}(V)=\frac{V f^{\prime}(V, \mathbf{W})-V^{2} f^{\prime \prime}(V, \mathbf{W})}{f(V, \mathbf{W})}, \\
P_{p}(V)=\frac{f(V, \mathbf{W})+0.5 V^{2} f^{\prime \prime}(V, \mathbf{W})-V f^{\prime}(V, \mathbf{W})}{f(V, \mathbf{W})},
\end{gathered}
$$

where $f^{\prime}(V, \mathbf{W})=\frac{\partial f}{\partial V}$ and $f^{\prime \prime}(V, \mathbf{W})=\frac{\partial^{2} f}{\partial V^{2}}$.

Various approaches exist for creating general nonlinear models of the power versus voltage load profile, such as high order polynomials, B-splines, SVMs and neural networks. In this instance, single hidden layer Multilayer Perceptron (MLP) neural networks are chosen as the model structure. These have universal function approximation capabilities and provide smooth fits to nonlinear functions. By employing appropriate training and cross-validation procedures the number of hidden 
layer neurons $\left(N_{h}\right)$ and network weights $(\mathbf{W})$ can be optimized. This choice of model is also known to handle discontinuities well. The MISO MLP with one hidden layer, sigmoidal activation functions in the hidden layer neurons and a linear activation function in the output layer is defined as

$$
y=\sum_{i=1}^{N_{h}} c_{i} \operatorname{sig}\left(\sum_{j=1}^{N_{I}} w_{i j} u_{j}+b_{i}\right)+d
$$

where $\left\{c_{i}, w_{i j}, b_{i}, d\right\}$ are the network weights, constituting the model parameters $\mathbf{W}$, and $\operatorname{sig}(x)=\frac{1}{1+\mathrm{e}^{-\mathrm{x}}}$ is the sigmoid function The Jacobian $\frac{\partial y}{\partial \boldsymbol{u}}=\left[\begin{array}{lll}\frac{\partial y}{\partial u_{1}} & \cdots & \frac{\partial y}{\partial u_{N_{I}}}\end{array}\right]^{\mathrm{T}}$ of the MLP is computed as

$$
\frac{\partial y}{\partial u_{p}}=\sum_{i=1}^{N_{h}} c_{i} w_{i p} \operatorname{sig}^{\prime}\left(\sum_{j=1}^{N_{I}} w_{i j} u_{j}+b_{i}\right)
$$

and the Hessian matrix $\mathbf{H}=\left[h_{p q}\right]$ of second derivatives $\frac{\partial^{2} y}{\partial u_{p} \partial u_{q}}$ is given by

$$
\frac{\partial^{2} y}{\partial u_{p} \partial u_{q}}=\sum_{i=1}^{N_{h}} c_{i} w_{i p} w_{i q} \operatorname{sig}^{\prime \prime}\left(\sum_{j=1}^{N_{I}} w_{i j} u_{j}+b_{i}\right)
$$

where the first and second derivatives of $\operatorname{sig}(x)$ are given by

$$
\begin{gathered}
\operatorname{sig}^{\prime}(x)=\operatorname{sig}(x)(1-\operatorname{sig}(x)) \\
\operatorname{sig}^{\prime \prime}(x)=\operatorname{sig}^{\prime}(x)(1-2 \operatorname{sig}(x))
\end{gathered}
$$

For the load characterization approach considered here, the MLP model reduces to

$$
f(V, \mathbf{W})=\sum_{i=1}^{N_{h}} c_{i} \operatorname{sig}\left(w_{i} V+b_{i}\right)+d
$$

and the corresponding Jacobian and Hessian matrix reduce to

$$
\begin{gathered}
f^{\prime}(V, \mathbf{W})=\sum_{i=1}^{N_{h}} c_{i} w_{i} \operatorname{sig}^{\prime}\left(w_{i} V+b_{i}\right) \\
f^{\prime \prime}(V, \boldsymbol{W})=\sum_{i=1}^{N_{h}} c_{i} w_{i}^{2} \operatorname{sig}^{\prime \prime}\left(w_{i} V+b_{i}\right)
\end{gathered}
$$




\section{RESULTS}

In this section, the methodology introduced in Section 3, Equations (8) to (16) in particular; is applied to estimate load models for: (a) a simulated load characteristic corresponding to a $5 \mathrm{k} \Omega$ resistance; (b) measurement data for a $60 \mathrm{~W}$ incandescent light bulb; and (c) measurement data for a modern 9 W LED light bulb. In all cases active power measurements are recorded for applied voltages in the range $-12 \%$ to $+14 \%$ of the nominal voltage of $230 \mathrm{~V}$ in increments of $2 \%$. The MLP networks are trained using the BFGS training algorithm [10] with leave-one-out cross validation used to optimize the number of neurons, $N_{h}$. The results obtained for the three loads are presented in rows (a), (b) and (c) of Fig. 2 and Fig. 3, respectively.

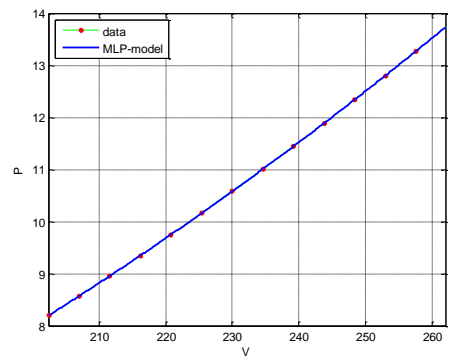

(a)(i) MLP model for $5 \mathrm{k} \Omega$ resistor

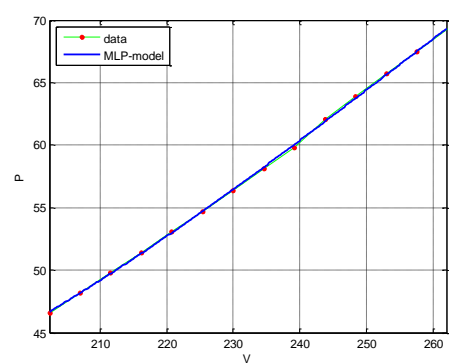

(b)(i) MLP model for Incandescent lamp

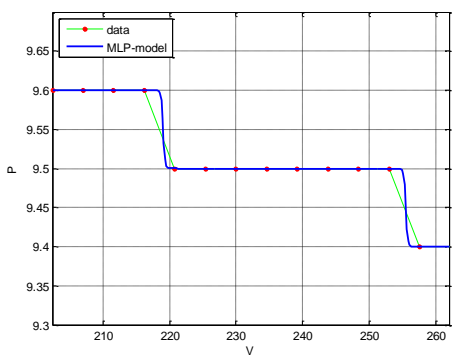

(c)(i) MLP model for LED lamp

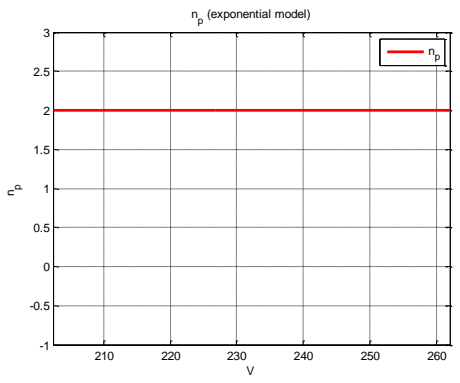

(a)(ii) $n_{p}$ for $5 \mathrm{k} \Omega$ resistor

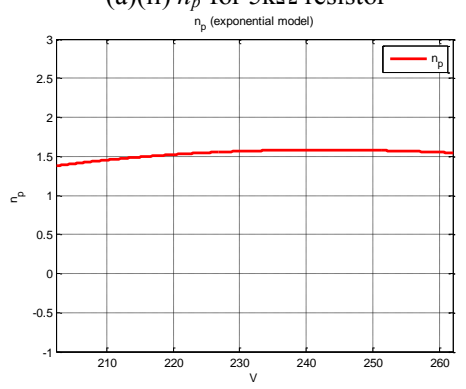

(b)(ii) $n_{p}$ for Incandescent lamp

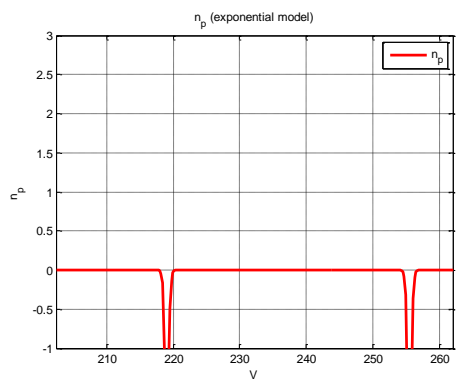

(c)(ii) $n_{p}$ for LED lamp

Fig. 2: Plots of the P-V data and MLP model approximation and small-signal exponential model estimates for each of the sample loads. 
In Fig. 2, the plots in the column denoted (i) show the MLP approximation of the P-V characteristic achieved, while plots of the small-signal exponential model coefficient, $n_{p}$, (computed according to (8)) are displayed in column (ii). In Fig. 3, the plots in column (i) show the small-signal ZIP parameter estimates determined directly from the MLP model; using (9), with column (ii) depicting plots of the small-signal ZIP parameter estimates determined indirectly from the $n_{p}$ estimate recovered in (5).

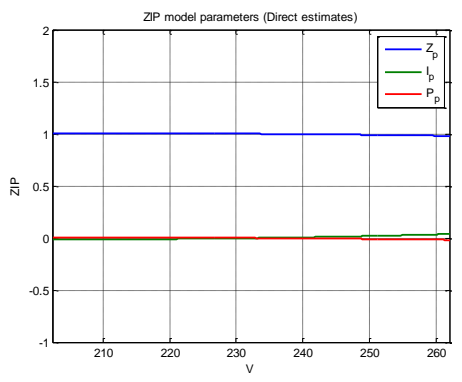

(a)(i) ZIP model parameters for $5 \mathrm{k} \Omega$ resistor (direct method)

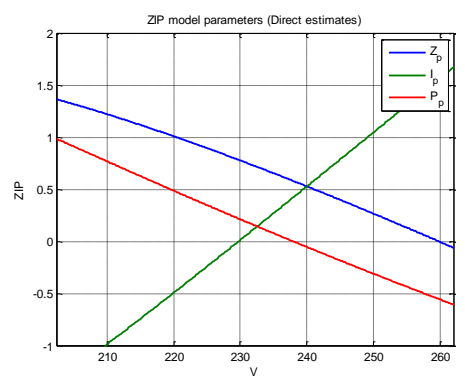

(b)(i) ZIP model parameters for Incandescent lamp (direct method)

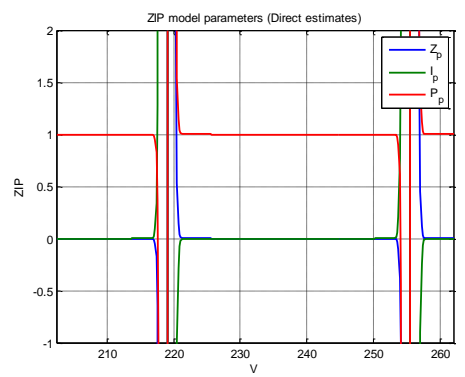

(c)(i) ZIP model parameters for LED lamp (direct method)

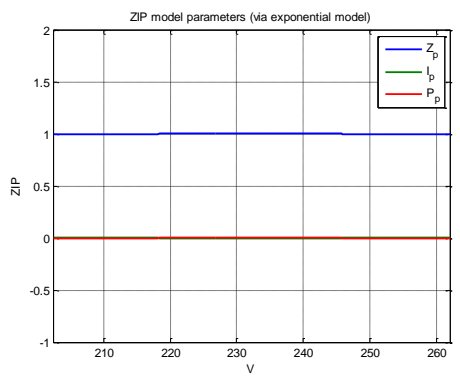

(a)(ii) ZIP model parameters for $5 \mathrm{k} \Omega$ resistor (indirect method, via $n_{p}$ )

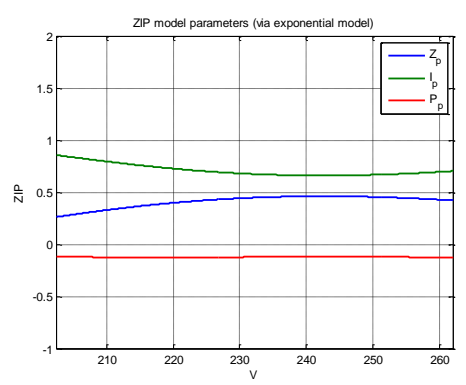

(b)(ii) ZIP model parameters for Incandescent lamp (indirect method, via $n_{p}$ )

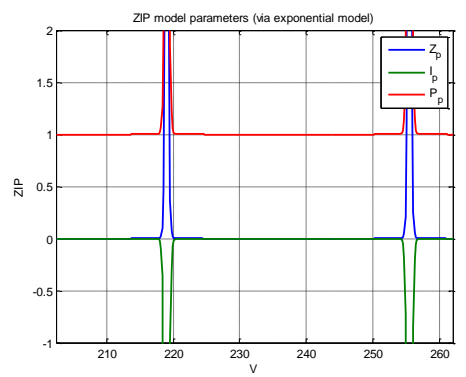

(c)(ii) ZIP model parameters for LED lamp (indirect method, via $n_{p}$ )

Fig. 3: Plots of small-signal ZIP parameters estimates (directly from MLP model); and small-signal ZIP parameter estimates, indirectly derived from the exponential model estimate for each of the sample loads 
As an ideal constant impedance example, the expected value of $n_{p}$ for the $5 \mathrm{k} \Omega$ resistor over all voltage levels is 2 , and the corresponding ZIP model values are $\mathrm{Zp}=1, \mathrm{Ip}=0$, $\mathrm{Pp}=0$. Fig. 2 confirms that the proposed methodology yields small-signal ZIP and exponential model parameter estimates consistent with these theoretical values. It is noteworthy that the indirect estimation of the ZIP parameters is more stable than direct estimation which is more susceptible to model approximation errors. This is a consequence of the extra degrees of freedom in the ZIP model and its reliance on local curvature information for parameter estimation.

The limitations of the direct small-signal ZIP model parameter estimation are even more apparent in Fig. 3, which shows the results for the incandescent light bulb. Here the stationary (global) exponential load model estimate is $n_{p}=1.54$ and the stationary (global) ZIP load model of the device is $Z_{p}=0.72, I_{p}=0.11, P_{\mathrm{p}}=0.17$. One might expect for this device to have a $n_{p}$ value of 2 . However, in practice the resistance of the bulb is strongly temperature dependent, and thus increases with applied voltage level, yielding a $n_{p}$ value closer to 1.5 . Comparing the stationary model coefficients with the small-signal variants, it is clear that the small-signal exponential model provides consistent estimates - varying between 1.38 and 1.58 with an average of 1.53 . In contrast, the directly estimated small-signal ZIP model parameters vary widely and do not correlate with the stationary ZIP model parameters. The indirectly estimated, smallsignal ZIP parameters are more stable, but it is noted that they differ substantially from the conventional, stationary model with mean values of $0.41,0.71$ and -0.12 for $Z_{p}, I_{p}$, and $P_{\mathrm{p}}$, respectively. This underscores the differences that exist between a single, stationary model covering the full device operating envelope and locally valid, smallsignal models.

Fig. 2(c)(i) shows how the LED bulb switches between three piece-wise, constant power intervals over the voltage range investigated. However, the conventional stationary ZIP model fit of this load profile, plotted in Fig. 1(a), does not adequately capture this behavior. It is clear that the MLP model provides a much superior fit to the load profile than the downwards sloping, close to linear approximation observed within Fig. 1(a). In addition, the small-signal exponential and ZIP models correctly identify the load as being constant power over most of the voltage range, except in the vicinity of the discontinuities, where they break down. 


\section{CONCLUSIONS}

This paper introduces a preliminary method for producing high accuracy, load model parameter estimates for modern load types. An MLP-based approach has been shown to offer a high fidelity fit to the P-V characteristics determined for the featured load types, from experimental measurements. This is particularly evident in the case of the piecewise functionality exhibited by an LED lamp in Fig. 2(c). Direct and indirect methods for small-signal, ZIP parameter estimates are then applied to the MLP generated trends. In each case the indirect approach is observed to offer superior performance. Fig. 3 (b)(ii) demonstrates how the indirect method is able to accurately track fluctuations in the underlying impedance of an archetypal, "constant" impedance load (incandescent lamp), as the applied voltage changes. This behavior, reflective of this load type's temperature dependency, is not reliably captured by conventional approaches, within which a stationary set of ZIP coefficients are typically determined across the entire voltage range.

\section{References}

1. Wang, Z., Wang. J.: Review on Implementation and Assessment of Conservation Voltage Reduction. IEEE Trans. Power Syst. 29(3), 1306-1315 (2014).

2. Singh, R., Tuffner, F., Fuller, J., Schneider, K.: Effects of Distributed Energy Resources on Conservation Voltage Reduction (CVR). In: IEEE Power and Energy Society General Meeting (2011).

3. Peskin, M., Powell, P., Hall, E.: Conservation Voltage Reduction with Feedback from Advanced Metering Infrastructure. In: IEEE PES Transmission and Distribution Conf. and Expo., Orlando, FL., (2012).

4. Nam, S-R., Kang, S-H., Lee, J-H., Ahn S-J., Choi J-H.: Evaluation of the Effects of Nationwide Conservation Voltage Reduction on Peak-Load Shaving Using SOMAS Data. Energies 6(12), 6322-6334 (2013).

5. Nam, S-R., Kang, S-H., Lee, J-H., Choi E-J., Ahn S-J., Choi J-H.: EMS-Data-Based Load Modeling to Evaluate the Effect of Conservation Voltage Reduction at a National Level. Energies 6(8), 3692-3705 (2013).

6. Hunt J.: Voltage optimization. IET Wiring Matters 47, 29-34 (2013).

7. IEEE Task Force on Load Representation for Dynamic Performance: Load Representation for Dynamic Performance Analysis. IEEE Trans. Power Syst. 8(2), 472-482 (1993).

8. IEEE Task Force on Load Representation for Dynamic Performance: Load Representation for Dynamic Performance Analysis. IEEE Trans. Power Syst. 10(3), 1302-1313 (1995).

9. Bokhari A. et al.: Experimental Determination of the ZIP Coefficients for Modern Residential, Commercial, and Industrial Loads. IEEE Trans. Power Del. 29(3), 1372-1381 (2014).

10. McLoone S., Brown M., Irwin G., Lightbody G.: A hybrid linear/nonlinear training algorithm for feedforward neural networks. IEEE Trans. Neural Netw. 9(4), 669-684 (1998). 